\title{
D-Transposition of great vessels with intact ventricular septum presenting at $3-8$ weeks: Should all go for rapid two stage arterial switch or primary arterial switch?
}

\author{
Akshay Kumar Bisoi, M.Ch. ${ }^{1}$, Sandeep Chauhan, MD. ${ }^{2}$ Sandeep Dilip Khanzode, MS. ${ }^{1}$, \\ Milind Padmakar Hote, M.Ch. ${ }^{1}$ Rajneesh Juneja, DM. ${ }^{3}$ Panangipalli Venugopal, M.Ch. ${ }^{1}$ \\ ${ }^{1}$ Department of Cardiovascular and Thoracic Sciences, ${ }^{2}$ Department of Cardiac Anaesthesia, ${ }^{3}$ Department of \\ Cardiology, All India Institute of Medical Sciences, New Delhi
}

\begin{abstract}
Background: Current reports favour primary arterial switch (ASO) in infants with d-transposition of great vessels (d-TGA) with intact ventricular septum (IVS) who present later than 21 days. The premise is that the regressed left ventricle $(\mathrm{LV})$ will still adapt to the systemic circulation.

Methods: We compared a retrospective group of 11 infants (group A) who had undergone rapid two stage ASO with those (group B) who had undergone primary ASO (n=15).

Results: The age range ( $25-70$ days), weight $(2.5-4.0 \mathrm{Kg})$, posterior wall thickness of $\mathrm{LV}(2.8 \mathrm{~mm}-4.2 \mathrm{~mm})$ and other pre-operative criteria were similar in both groups. In group A, 3/11 infants died after first stage, one each due to shunt blockade, tight pulmonary artery band, and after emergency Senning operation following cardiac failure. Of the remaining eight who underwent ASO 5 - 9 days after first stage, three died, one each due to fungal infection, sepsis and multi-organ failure, and massive haemorrhage from internal mammary artery. Success of LV training was 8/11 (73\%) while overall survival was 5/11 (45\%). In group B (15 infants), 13 survived $(86 \%)$, two needing post-operative extracorporeal membrane oxygenator (ECMO) support and two deaths occurred due to immediate post-operative cardiac failure.

Conclusions: This study demonstrates that primary ASO can show benefit in infants of d-TGA with IVS presenting between 21 to 60 days of age as compared to rapid two stage ASO. These infants might need more support for the ventriclular function in form of prolonged inotropes and ECMO support. (Ind J Thorac Cardiovas Surg 2006; 22: 5-9)
\end{abstract}

Key words: d-Transposition of great vessels, Intact ventricular septum, Arterial switch operation

Introduction

Left ventricular (LV) regression is the critical issue in infants with d-transposition of great vessels (d-TGA) with an intact ventricular septum (IVS). A regressed LV is unable to sustain the systemic circulation ${ }^{1}$. The option which remains is to accept an inferior procedure like atrial switch or adopt LV training techniques and attempt the superior arterial switch operation (ASO).

Address for correspondence:

Akshay Kumar Bisoi

Additional Professor,

Department of Cardiothoracic and Vascular Surgery,

CN Centre, $7^{\text {th }}$ floor, All India Institute of Medical Sciences,

Ansari nagar, New Delhi - 110 029, India.

(C) IJTCVS 097091342210305/76

Received - 30/10/05; Review Completed - 18/11/05; Accepted - 16/12/05.
Traditionally it has been accepted that upto 21 days of age, LV can sustain the systemic circulation. Hence ASO is offered to neonates of d-TGA, IVS who are less than 21 days old $^{2}$. However, recent reports have suggested to extend this limit to two months of age $\mathrm{e}^{1,3,4}$. We retrospectively reviewed two groups of infants of dTGA with IVS who presented after 21 days of age. One group had been subjected to rapid LV training followed by ASO and other group had undergone primary ASO.

\section{Methods}

We evaluated the records of 11 infants who underwent the rapid two stage protocol (group A) and 15 infants of primary ASO group (group B). All these infants had been operated at All India Institute of Medical Sciences, New Delhi in between December 2003 to September 2005. Both these groups were analyzed 
on the basis of age, echocardiographic findings of adequacy of atrial septal defect (ASD), coronary artery pattern, relationship of great vessels, presence of patent ductus arteriosus (PDA) and LV preparedness.

\section{Surgical technique and post-operative care}

Group A infants underwent an innominate artery to right pulmonary artery (RPA) shunt with polytetraflouroethylene graft of $3.5 \mathrm{~mm}$ through standard median sternotomy. Simultaneous main pulmonary artery band (PAB) with mersilene tape. No attempt was made to wean the neonate off ventilator after the first stage. In case of any sudden deterioration, the cause was identified and either band was loosened or shunt thrombectomy carried out as needed. Serial echocardiographic examinations were carried out to look for adequate band gradient, LV cavity dimensions, LV posterior wall and septal thickness. Infants who survived the first stage were taken up for the second stage as soon as LV seemed prepared. The shunt was taken down, PAB was removed, and ASO was performed. Cardiopulmonary bypass was established using aortic and single right atrial cannulation. The ASD was closed on a period of circulatory arrest of less than five minutes. Group B infants were taken for primary ASO with cardiopulmonary bypass (CPB) established using a bicaval cannulation. The surgical technique was similar to what adopted in group A. Except that the ASD was closed on CPB without circulatory arrest. Postoperative care in form of inotropes, vasodilators, was used in all cases, and adequate urinary output, peripheral temperature, and absence of high core temperature were targeted. Acidosis in blood gases was avoided. Use of peritoneal dialysis, additional inotropic support, and extracorporeal membrane oxygenation was undertaken whenever felt necessary. Infants were weaned off ventilator after haemodynamic stabilization. Gradually inotropic support was withdrawn the children were shifted out of intensive care unit.

\section{Statistical Analysis}

Outcomes between groups were compared using non-paired two tailed student $t$ test for continuous variables. Discrete variables were compared using Fisher's exact chi square test. Statistical softwares used were Microsoft ${ }^{\circledR}$ Excel 2002 and EpiInfo ${ }^{\text {TM }} 2002$ computer programs.

\section{Results}

A total of 150 infants underwent ASO, 70 with d-TGA, IVS at All India Institute of Medical Sciences, New Delhi, India from January 2003 to September 2005. 40 infants of 70 presented later than 21 days of age. 16 infants underwent rapid two stage ASO performed by one surgeon at the institute. 11 of 16 infants belonged to age between 30 days to 70 days. Rest five infants were more than 4 month old and were excluded. Thus group A included 11 infants who had undergone rapid two stage ASO. Of the rest 24 infants, 15 infants operated by a single separate surgeon were included as group B. The age of these 15 infants ranged between 30 days to 60 days. Both the groups were comparable with respect to age $(\mathrm{P}$ value $=0.12)$.

Table 1 includes details of pre-operative characteristic in these infants. One infant in group B had left ventricular outlet obstruction with gradient of $18 \mathrm{~mm}$ $\mathrm{Hg}$. None of these infants had any aortic arch issues, small muscular ventricular septal defects, or any other anatomic variation. Coronary artery pattern was usual $1 \mathrm{AD}, \mathrm{Cx} ; 2 \mathrm{R}$ according to Leiden classification in most patients and either $1 \mathrm{AD} ; 2 \mathrm{R}, \mathrm{Cx}$ or $1 \mathrm{R}, \mathrm{AD} ; 2 \mathrm{Cx}$ in the rest. None of these infants had intramural coronaries. Initial echocardiogram suggested a regressed left ventricle in seven infants of group A and B both.

Table 1. Pre-operative characteristics of the infants of groups A and $B$

\begin{tabular}{lrrr}
\hline Parameter & $\begin{array}{r}\text { Group A (n=11) } \\
\text { (Rapid 2 stage ASO) }\end{array}$ & $\begin{array}{r}\text { Group B (n=15) } \\
\text { (Primary ASO) }\end{array}$ & P value \\
\hline Age (days) & $30-70($ mean 49$)$ & $25-60($ mean 40$)$ & $0.12(\mathrm{~N} / \mathrm{S})$ \\
Patent Ductus & $7 / 11$ & $9 / 15$ & $1.0(\mathrm{~N} / \mathrm{S})$ \\
Atrial Septostomy & $7 / 11$ & $9 / 15$ & $1.0(\mathrm{~N} / \mathrm{S})$ \\
$\begin{array}{l}\text { Regressed LV } \\
\text { Posterior wall }\end{array}$ & $7 / 11$ & $7 / 15$ & $0.7(\mathrm{~N} / \mathrm{S})$ \\
$\begin{array}{l}\text { thickness } \\
\begin{array}{l}\text { Septal motion } \\
\text { with LV }\end{array}\end{array}$ & & & \\
\hline
\end{tabular}

11 infants underwent first stage in form of a pulmonary artery banding and innominate to right pulmonary artery shunt. Out of the 11 group A infants three deaths occurred after first stage. In one infant the shunt was blocked and needed a re-exploration. Subsequently the shunt got blocked again and the infant arrested and could not be revived. Infant 2 had sudden cardiac arrest, immediate loosening of pulmonary band was done at bedside, shunt was patent but the infant could not be resuscitated. The third infant went gradually into low cardiac output, left ventricular failure and acidosis. This infant was taken for emergency Senning procedure, but the infant died in immediate post-operative period. Only eight infants had a satisfactory LV preparation within five to nine days interval and underwent ASO as a second stage procedure. There were three deaths after second stage. 
One infant had a good recovery, but on $21^{\text {st }}$ postoperative day the ventricular pacing wire hit the left internal mammary artery from beneath and the infant had massive haemorrhage. The infant exsanguinated within no time and could not be salvaged despite immediate reexploration. One infant also had a satisfactory recovery, but had fulminant respiratory fungal infection and died after a month. The third infant had initial deterioration of ventricular function that gradually improved; however the prolonged course led to renal failure, severe nosocomial sepsis and death on $22^{\text {nd }}$ post-operative day. The average hospital stay for those who survived was $30-40$ days.

Two deaths occurred in group B. Both the deaths were in the initial post-operative period due to severe left ventricular failure. Of the thirteen infants who survived, two infants needed ECMO support $24-48$ days. One infant had low cardiac output in the postoperative period. ECMO support was established within four hours of surgery. The ventricular function improved and ECMO was weaned off in 48 hours. ECMO support was established in another infant just after cardiopulmonary bypass was weaned off as he had low systemic pressures. The ECMO support lead to early recovery of ventricular function and was weaned off ECMO within 24 hours. The average hospital stay in nine patients was $10-12$ days. Two infants required a tracheostomy which increased their post-operative stay for a month. They were discharged after weaning them off from tracheostomy. Figure 1 shows the overall outcome of both the groups.

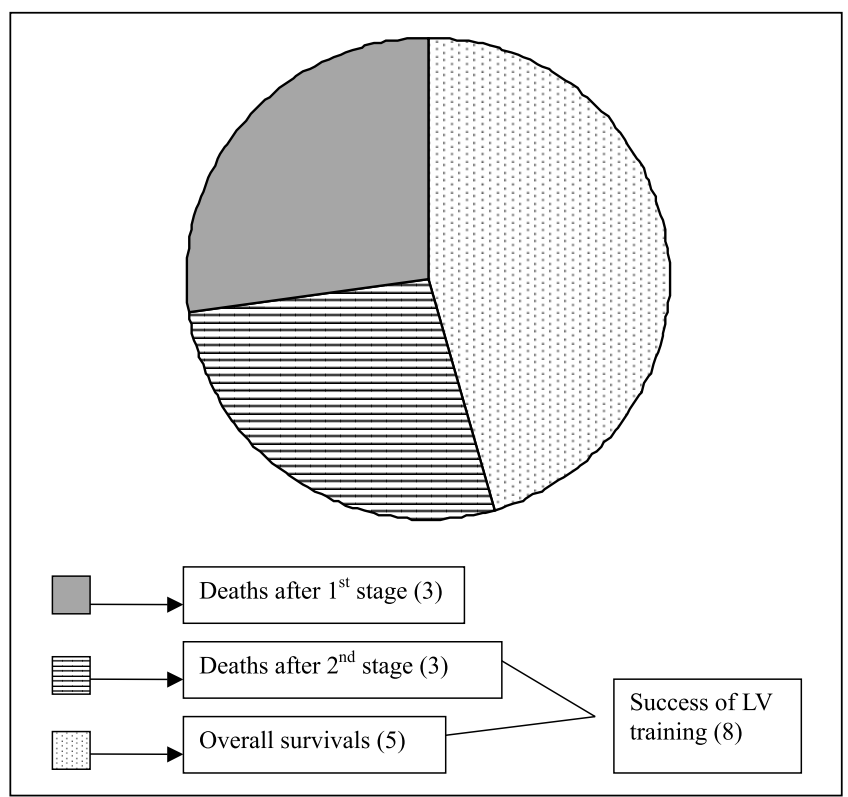

Fig.1. Outcome of Group A ( rapid 2 stage ASO)

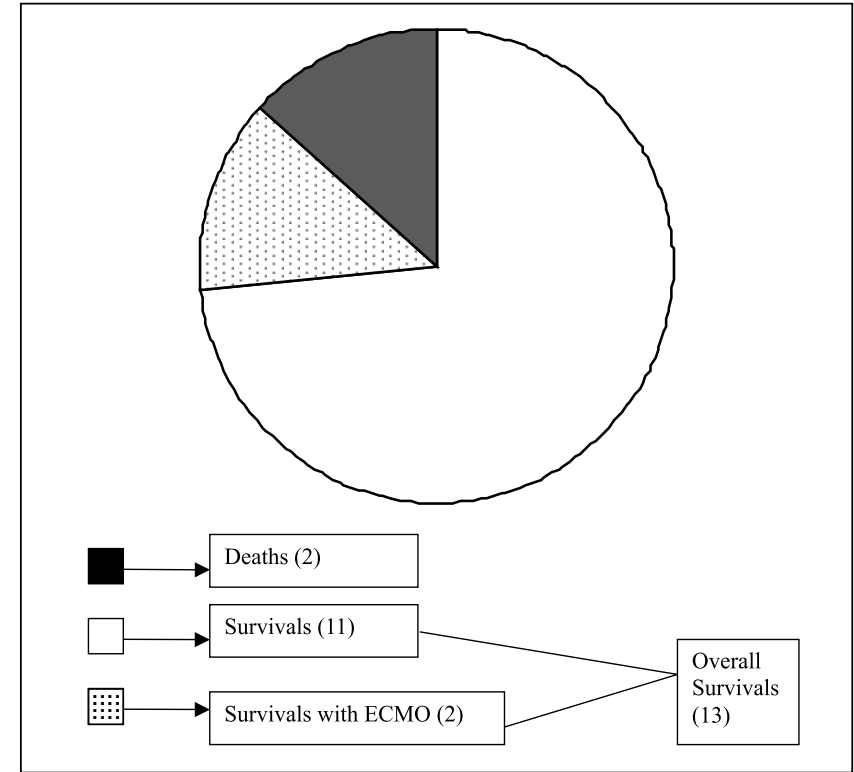

Fig.2. Outcome of Group B ( Primary ASO)

\section{Discussion}

The surgical management of transposition of the great arteries and intact ventricular septum (TGA/IVS) beyond 2 to 3 weeks of age is controversial. Concern that regression of the left ventricular (LV) myocardial mass will render the left ventricle incapable of coping with the acutely increased work of systemic perfusion has been considered a contraindication to a primary $\mathrm{ASO}^{3,5}$. Options available after $\mathrm{LV}$ regression are either atrial switch procedures like Senning or Mustard operation or left ventricular training followed by arterial switch ${ }^{1}$. The results of ASO had been encouraging as compared to the atrial switch operations. Worldwide it was accepted that ASO is superior to Senning/Mustard operations ${ }^{1}$. The 2 -stage arterial switch, which had been proposed a decade earlier, ${ }^{6}$ and later modified by reducing the interval between stage to 1 week, ${ }^{7}$ was put forward as a solution to infants presenting for surgery beyond the neonatal period ${ }^{7,8}$.

Proponents of staged arterial switch feel that infants with late referral have perinatal complications and LV dysfunction. It is felt that rapid LV training leads to LV remodeling as a result of increased $\mathrm{LV}$ afterload, LV wall shear stress and LV volume overload. This remodeling improves the performance of LV to adapt to systemic circulation. Many centers have tried the rapid two stage ASO protocol for such late referrals with variable success ${ }^{7,8}$. Echocardiography has emerged as an important tool for assessment of LV preparation ${ }^{8,9}$. LV mass index, LV posterior wall thickness, LV end- 
diastolic internal diameter, LV cavity shape, interventricular septal thickness and movement in coordination with either of ventricles were recognized as criteria that aid in the assessment ${ }^{8,9}$. However rapid two stage ASO has several issues that remain unclear and confound the overall outcome like size of shunt, tightness of band, defining preparedness, interval, morbidity and mortality, prolonged ICU stay leading to nosocomial infections and hospital costs. Furthermore, low cardiac output, systemic congestion with severe right heart failure is not uncommon as time elapses between first and second stage. At the same time reports have also shown that these infants have late $\mathrm{LV}$ systolic dysfunction due to increased afterload leading to decreased contractility ${ }^{10}$. Also they show increased incidence of neo-aortic regurgitation and right ventricular outflow obstruction in comparison to those who undergo primary ASO ${ }^{1}$.

In the group A, based on LV posterior wall thickness, LV systolic function, septal movement and LV cavity shape those infants who had a borderline or regressed $\mathrm{LV}$ underwent rapid two stage ASO. The tolerance of first stage is also a major concern as the first stage puts lot of strain on the ventricle. Potential problems can arise of tightness of PA band and overflow/blockage of the shunt. In case of any such event, infant life is jeopardized. This is evident from the $27 \%$ mortality $(n=3)$ after the first stage in our series. In the event of infants undergoing second stage, five of eight infants survived. But all the other three cases did not have LV dysfunction and LV failure and died of non-cardiac causes (2) and rare unusual cause (1). Thus the overall success of LV training followed by ASO is better (8/11, $73 \%$ ) if ventricular function and cardiac output is measured. The total survival however was only 5/11 infants (45\%). Thus overall the benefits of this protocol are doubtful in comparison with long term results of atrial switch procedure which have a very low operative risk and mortality ${ }^{1}$.

In the second group of infants who underwent primary ASO, 13 of 15 infants survived giving even better outcome from primary ASO. This suggests that despite of having borderline or regressed $L V$, the ventricle performed better as the systemic ventricle. All infants could be weaned off cardiopulmonary bypass after surgery, but in ICU showed features of low cardiac output after a period of two - four hours. These infants might require additional mechanical support in form of ECMO. The authors feel that the two infants who died could also be salvaged if timely ECMO support had been established. Thus they feel the need of predicting the need of ECMO support on the basis of pre-operative findings and also the timing of establishing it. Because excessive delay might lead to ventricular damage irreversible to effect of any intervention. ECMO support unfortunately results into higher overall costs and might be of concern in developing countries where majority of infants present at late age.

In support to the above findings, multiple series have shown success with primary ASO in such late referrals $^{1,3,4}$. Davis et al reported 18 infants with age $>$ than 21 days undergoing primary ASO with only one death $^{4}$. Kang et al have published the largest series of primary ASO in infants $>21$ days and compared them with outcome of ASO in infants $<21$ days. They found only $3.8 \%$ operative mortality in "late switch" group. They have also reported the need of ECMO support in such infants ${ }^{1}$. Duncan et al have reported eight infants (age of 15 - 46 days) with primary ASO with no mortality ${ }^{11}$.

In contrast is the report by Lacour-Gayet et al who advocate staged ASO if age $>$ than 21 days $^{8}$. Boutin et al also advocate rapid two stage ASO in case of delayed referrals. Their success with LV training and subsequent ASO has been fairly good ${ }^{10}$. An older study from our institute also reported success in 14 of 17 infants with mean age 4 months who underwent rapid two stage $\mathrm{ASO}^{9}$. Corno et al also describe success with LV preparation followed by ASO in 6 infants with age between $3-25$ months ${ }^{12}$.

The authors probably feel that instead of a definite cut off of 3 weeks in deciding the plan of action, a grey zone exists where alternative approach might yield better results. Recent reports suggest "extending the limits" of primary ASO to infants older than 21 days ${ }^{1}$.

\section{Conclusion}

In consideration with our review of infants with dTGA and IVS with age ranging between 21 days -2 months who underwent rapid two stage ASO and primary ASO, we feel that primary ASO can still give better results. The LV can sustain the systemic circulation without any evidence of failure. Support in form of ECMO can improve the results in infants who have LV failure in post-operative period.

\section{References}

1. Kang N, de Leval MR, Elliott M, Tsang V, Kocyildirim E, Sehic I, et al. Extending the boundaries of the primary arterial switch operation in patients with transposition of the great arteries and intact ventricular septum. Circulation. 2004; 110: 123-27.

2. Kirklin JW, Blackstone EH, Tchervenkov CI, Castaneda AR. Clinical outcomes after the arterial switch operation for 
transposition. Patient, support, procedural, and institutional risk factors. Congenital Heart Surgeons Society. Circulation 1992; 86: 1501-15.

3. Foran JP, Sullivan ID, Elliott MJ, de Leval MR. Primary arterial switch operation for transposition of the great arteries with intact ventricular septum in infants older than 21 days. J Am Coll Cardiol. 1998; 31: 883-89.

4. Davis AM, Wilkinson JL, Karl TR, Mee RB. Transposition of the great arteries with intact ventricular septum. Arterial switch repair in patients 21 days of age or older. J Thorac Cardiovasc Surg. 1993; 106: 111-15.

5. Norwood WI, Dobell AR, Freed MD, Kirklin JW, Blackstone EH. Intermediate results of the arterial switch repair. A 20-institution study. J Thorac Cardiovasc Surg. 1988; 96: 854-63.

6. Yacoub MH, Radley-Smith R, Maclaurin R. Two-stage operation for anatomical correction of transposition of the great arteries with intact interventricular septum. Lancet. 1977; 1: 1275-78.

7. Jonas RA, Giglia TM, Sanders SP, et al. Rapid two-stage arterial switch for transposition of the great arteries and intact ventricular septum beyond the neonatal period. Circulation. 1989; 80: I203-08.
8. Lacour-Gayet F, Piot D, Zoghbi J, Serraf A, Gruber P, Mace L, et al. Surgical management and indication of left ventricular retraining in arterial switch for transposition of the great arteries with intact ventricular septum. Eur J Cardiothorac Surg. 2001; 20: 824-29.

9. Iyer KS, Sharma R, Kumar K, Bhan A, Kothari SS, Saxena A, et al. Serial echocardiography for decision making in rapid twostage arterial switch operation. Ann Thorac Surg. 1995; 60: 65864.

10. Boutin C, Wernovsky G, Sanders SP, Jonas RA, Castaneda AR, Colan SD. Rapid two-stage arterial switch operation. Evaluation of left ventricular systolic mechanics late after an acute pressure overload stimulus in infancy. Circulation. 1994; 90: 1294-303.

11. Duncan BW, Poirier NC, Mee RB, Drummond-Webb JJ, Qureshi

A, Mesia CI, et al. Selective timing for the arterial switch operation. Ann Thorac Surg. 2004; 77: 1691-96; discussion 1697.

12. Corno AE, Hurni M, Payot M, Sekarski N, Tozzi P, von Segesser LK. Adequate left ventricular preparation allows for arterial switch despite late referral. Cardiol Young. 2003; 13: 49-52. 